\title{
Experiment of Wireless Tele-echography System by Controlling Echographic Diagnosis Robot
}

\author{
Kohji Masuda, Norihiko Tateishi, Yasuyuki Suzuki, \\ Eizen Kimura, Ying Wie, and Ken Ishihara \\ Department of Medical Informatics, Ehime University Hospital, \\ Shigenobu-cho, Onsen-gun, Ehime 791-0295 Japan \\ masuda@vishnu.m.ehime-u.ac.jp \\ http://www.medinfo.m.ehime-u.ac.jp/
}

\begin{abstract}
We have developed a wireless tele-echography system for controlling an echographic diagnosis robot (EDR) that was developed in our laboratory. The examiner can control the robot at a distance from the patient and obtains both an image of the patient's status and an echogram of the internal organs. The locations of the examiner and the patient are connected by a wireless network. Remote control was realized by means of the Object Request Broker technique. By this experiment, we have thus verified the usefulness of our system for clinical practice. Remote diagnosis of an echogram with sufficient image quality was possible after a skilled examiner had become used to controlling the robot. The adult patient never felt any discomfort or pain from the robot. Since the maximum bandwidth required by this system was $1 \mathrm{Mbps}$, we have confirmed that wireless tele-echography can become a reality in telemedicine and telecare.
\end{abstract}

\section{Introduction}

We have developed an echographic diagnosis robot[1,2] to control an ultrasound probe. The examiner captures echograms by placing the robot on the body surface of a patient. The robot is designed to move the probe on the human abdomen in three dimensions, has 6 degrees of freedom of movement, detects contact force on the body and encompasses carefully considered safety measures. Although several groups have previously developed mechanisms to handle an ultrasound probe, almost all of these are of the manipulator type [3,4] and involve a large system [5]. In applying such manipulator robots to medical needs, position control is difficult to achieve when the body motion of the patient has to be followed. Thus, we considered that the entire mechanism for moving the ultrasound probe should be lightweight and be placed on the body itself [6].

We have already tried a preliminary experiment involving tele-echography via the Internet and an ISDN network [1,2]. Just a few years ago, the bandwidth of a popular ISDN network was mostly $128 \mathrm{kbps}$ in Japan. Surprisingly, there has been rapid progress in line speed, and ADSL at $1 \mathrm{Mbps}$ became available to private users quite recently. Thus, we considered the feasibility of extending the tele-echography system rather than adhering to line speed. In this paper, we describe a preliminary experiment 
in wireless tele-echography involving the control of our echographic diagnosis robot in order to verify the possibility of clinical use.

\section{Wireless Tele-echography System}

\subsection{Overview}

Fig. 1 shows the concept of our wireless tele-echography system. An examiner is located quite separate from the patient and controls the echographic diagnosis robot which has been placed on the abdomen of the patient. The ultrasound probe is held by the robot and it moves on the abdomen under the control of the examiner. The patient status images and echograms are transferred back to the examiner. The patient and examiner can communicate by seeing and talking to each other.

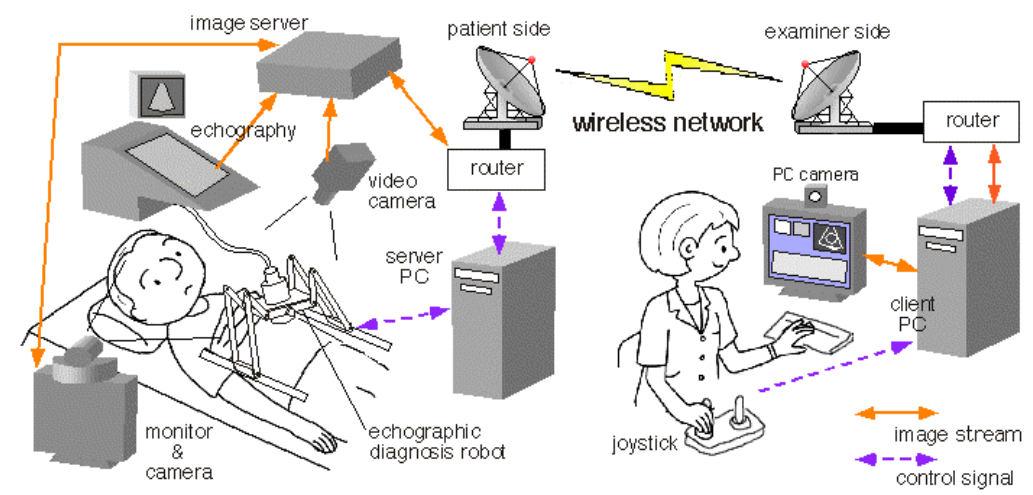

Fig. 1. Scheme of wireless tele-echography system

The most recent internet applications are moving towards wireless and mobile. Wireless network technology is very useful for telemedicine, especially in Ehime district, as there is no need to run wires and cables. Ehime prefecture includes both high mountains and isolated islands, which are typical Japanese geographical features. Although the present accessibility distance is several kilometers at most, international connection might be possible in the future. Wireless networks can be established quickly at low cost, which enables the possibility of setting up a temporary clinic in the case of a large-scale disaster.

We used two wireless LAN bridges SB-1100 (ICOM Co. Ltd.). One is included in the ordinary LAN in Ehime University which we usually use at $10 \mathrm{Mbps}$. The other is used as a temporary network with a router and notebook PC in a temporary examination room located separately from Ehime University Hospital.

Fig. 2 shows the map of Shigenobu-cho(town) in Ehime prefecture, Japan. We selected a temporary examination room in the Twin Dome Shigenobu which is located $1.4 \mathrm{~km}$ from our hospital. There is no obstruction for wireless telegraphy between the Twin Dome and the hospital. In this experiment, we established the examiner site in 
the Twin Dome because the echographic diagnosis robot is complicated in wiring and power supply at present, and is difficult to remove from the hospital.

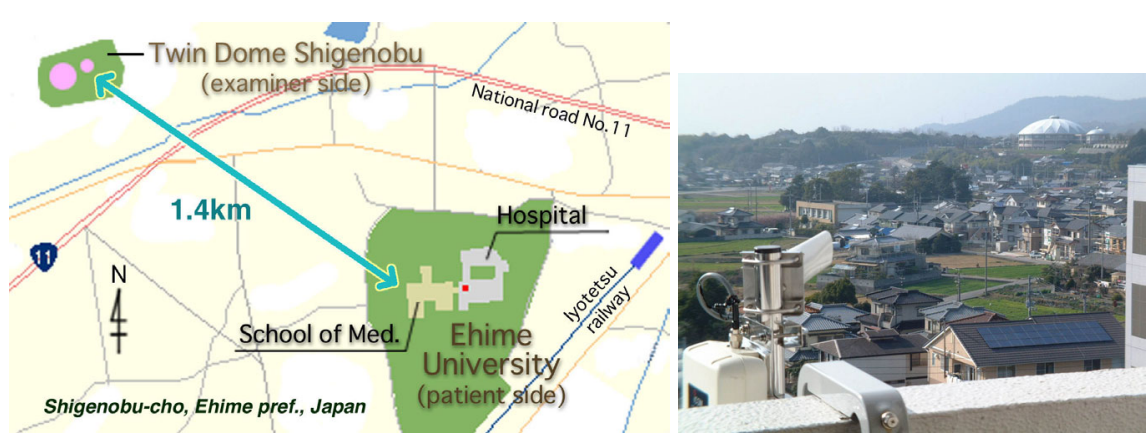

Fig. 2. Geographical situation. Map of Shigenobu-cho (left). The distant view of Twin Dome Shigenobu from Ehime University with wireless network antenna (right)

\subsection{Situation at the Patient Location with Echographic Diagnosis Robot}

Fig. 3 shows the situation at the patient site. The image streaming server (PCS-1600, Sony Co. Ltd.) is on the monitor beside the patient, as shown in Fig. 3 (a). It captures not only two patient status images from two video cameras, but also echograms captured by echography (EUB-565S, Hitachi Medical Co. Ltd.) and encodes this into the image stream. The examiner is able to switch among these images via the network.

The echographic diagnosis robot, which we have developed to realize 6 DOF motion of three-dimensional rotation and translation, moves according to the command from the examiner. Precise mechanism is shown in Fig. 3 (b). The echographic diagnosis robot is placed on the abdomen of the patient. Its total weight is $3.3 \mathrm{~kg}$, which is insufficient for adults to feel heavy.

The robot consists of gimbal, pantograph and slide mechanisms. The gimbal mechanism tilts the probe by three independent motors. The probe is fixed in an acrylic resin column which has a brim where four miniature force sensors are located, to detect contact forces against the body surface as shown in Fig. 3 (c). The reason for using four sensors is as follows. Since a summation of output of four sensors reveals the total contact force on the body, the pantograph motion can be limited so that it will not push the body by strong force. Furthermore, the difference in output between two symmetrical sensors indicates the angle of the probe to the body surface. Thus, safety control is possible by computing the output of four sensors so that dangerous physical positions can be avoided [1].

Both the pantograph and the slide mechanisms effect translation of the probe on the abdomen. Parallel $(x)$ and rectangular $(y)$ translation of the probe are done by moving actuators located at the bottom of the pantograph. Two slide actuators are set on the abdomen to move the pantograph mechanism in the body axis direction. Each side of the slide effects linear motion by a linkage between two joints.

Since the bottom of the pantograph mechanism is put on the side of the abdomen, the arms of the pantograph never harm the patient's body. Fig. 3 (d) shows the geometry of the pantograph mechanism. Defining the positions of the slide actuators as 
$(0,0)$ and $(d, 0)$ in rectangular coordinates and the intersection point of the gimbal axis as $P\left(x_{p}, y_{p}\right)$ shown in Fig. 3(d), while either of the angles of motors $\theta_{1}$ and $\theta_{2}$ do not exceed $\pi / 2$, the coordinates of $P\left(x_{p}, y_{p}\right)$ can be expressed as follows.

$$
\begin{gathered}
x_{p}=\frac{a\left(\cos \theta_{1}-\cos \theta_{2}\right)+d}{2} \\
y_{p}=a\left(\sin \theta_{1}+\sin \theta_{2}\right) / 2 \\
-\frac{1}{2} \sqrt{4 b^{2}-(a-c)^{2}+2 a(a-c)\left(\cos \theta_{1}+\cos \theta_{2}\right)-2 a^{2}\left\{1+\cos \left(\theta_{1}+\theta_{2}\right)\right\}}
\end{gathered}
$$

In these equations, $a, b$ represent the arm lengths of the pantograph, $c$, the width of the gimbal and $d$, the distance between two slides, respectively.

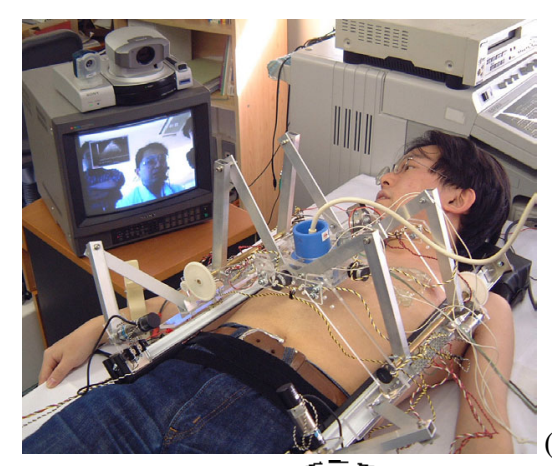

(a)

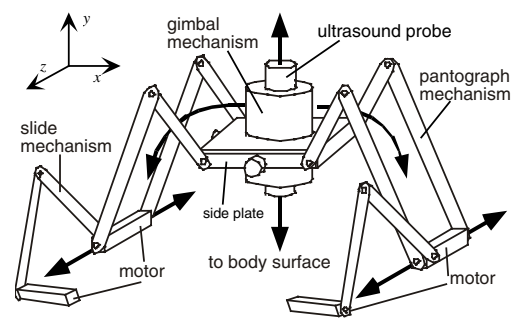

(b)
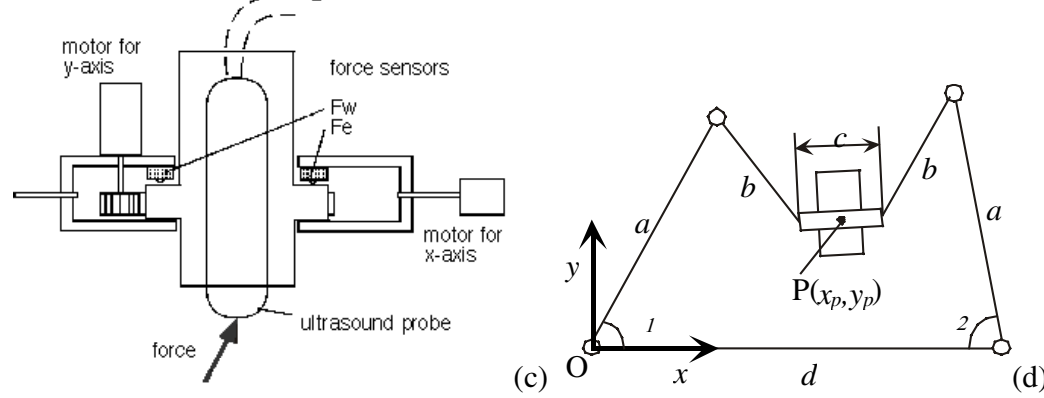

Fig. 3. Situation at patient location. (a) Overall view of echographic diagnosis robot on human abdomen. (b) Precise mechanism of the robot. (c) Cross-section of the gimbal mechanism. (d) Geometry of the pantograph mechanism

\subsection{Situation at Examiner Location with Human Interface}

Fig. 4 shows the scene at the examiner site. A skilled medical doctor controls the remote robot by a two-axis joystick (Compact analog stick, Hori Co. Ltd.). The control interface was designed for the examiner in the way of observing the patient from the direction of the feet. The method of control is shown to the right of Fig. 4. The angle of the right stick corresponds completely to the angle of the gimbal mechanism. Rotation of the probe axis is done by pushing two buttons with the right forefinger. The 
left stick is used for pantograph motion. After adjusting the initial position and gain, which depend on the body size of the patient, the angle of the left stick corresponds to the coordinates of the probe position $P\left(x_{p}, y_{p}\right)$. Slide motion in body axis is made by pushing two buttons with the left forefinger.
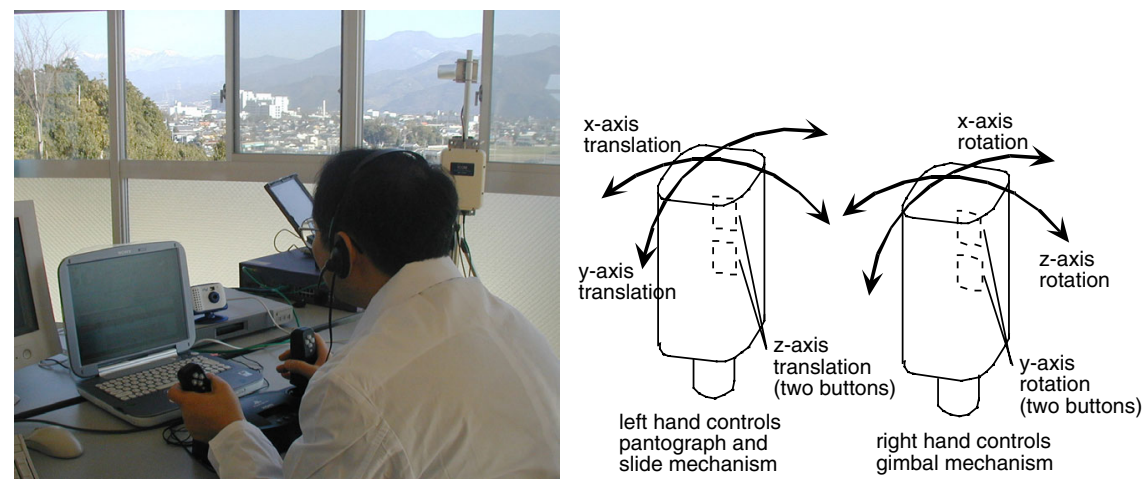

Fig. 4. Situation at examiner location. The examiner controls the remote robot by two-axis joystick while observing the patient status on a PC monitor. Ehime University Hospital can be seen out of the window (left). Method of controlling the robot by the joystick (right)

The examiner inspects the echogram and the patient status image simultaneously on the PC monitor, as shown in Fig. 5. The upper right window is the echogram, the upper middle is a robot image to confirm the position of the probe, and at the upper left is the examiner's own image which is sent to the patient. The upper right window can be changed regarding input source. The lower window is for input selection and the control of camera angle. Voice communication between the examiner and the patient is also possible.

\subsection{Object Request Broker}

To control the robot via a network, we applied the HORB [7] technique, which is derived from ORB (Object Request Broker) technique in distributed object systems. HORB satisfies higher speed transfer and better facility of development compared to CORBA [8]. We developed the software under the C++ and Java platforms [9].

\section{Results of Experiment}

We prepared to conduct the experiment over two days in February. The first day was rainy and it snowed intermittently. The next day was very fine. On both days, the line speed was sufficient to enable communication at up to $6 \mathrm{Mbps}$ bandwidth, confirming that the weather was not important for wireless communication. The robot followed the command from the examiner and touched closely on the surface of abdomen. We adopted ITU T.120 standard data protocols with ITU G.728 standard voice compression, which enabled high quality conference conditions with real-time moving echo- 
gram. The time delay was less than $1 \mathrm{sec}$ when image size was CIF 352 x 288 pixels for the echogram at 512Kbps and QCIF $176 \times 144$ pixels for the patient status image at $100-200 \mathrm{Kbps}$. This was much more comfortable than our previous experiment $[1,2]$ where we used motion-JPEG encoding and the time delay was 4-5 sec, which was very stressful.
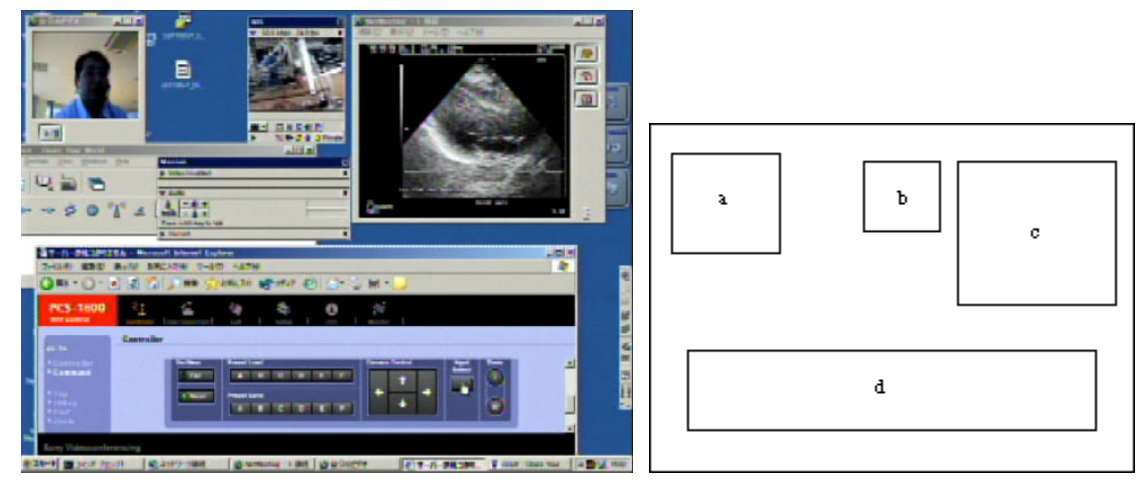

Fig. 5. PC monitor at the examiner location. (a) examiner's own face, (b) patient status, (c) echogram and (d) video camera control with input selection. The examiner's facial image is transferred to the patient location. Two kinds of patient status image and the echogram can be switched as desired by the examiner

The examiner got used to operating the controls after a few minutes of practice. The quality of the echogram was sufficient for diagnosis. Communication with the patient was as easily achieved as good-quality telephone link. When observing the heart, the examiner had no difficulty in instructing the patient to stop breathing and to resume.

The adult patient did not feel any discomfort after the robot had been put on the abdomen, and even after an experiment lasting more than one hour. Only the slide part touched to the body (after adjusting the width of the slide according to the body size). However, we do not know whether this design would be for a pregnant woman or an infant. Thus, for clinical use, several different types of robot construction should be prepared to suit different patients.

Before the experiment, we thought that one patient image would be enough on the examiner's monitor because the examiner can switch between echogram and patient status images as desired. However, if the examiner observed the echogram alone, the direction of ultrasound probe on the body surface became confused. To avoid stress, at least two kinds of image are necessary on the PC monitor at the same time.

Fig. 6 shows the variation in packet number and bit rate which passed through the wireless LAN bridge during the experiment. The horizontal line expresses time during the experiment. Coloured areas express packet number of image stream and control signal, respectively, which are designated on the left axis. The solid line expresses total bit rate, which is designated on the right axis. Total bit rate was at most $1 \mathrm{Mbps}$, so that this system can be accepted into the infrastructure of recent fast digital networks. 


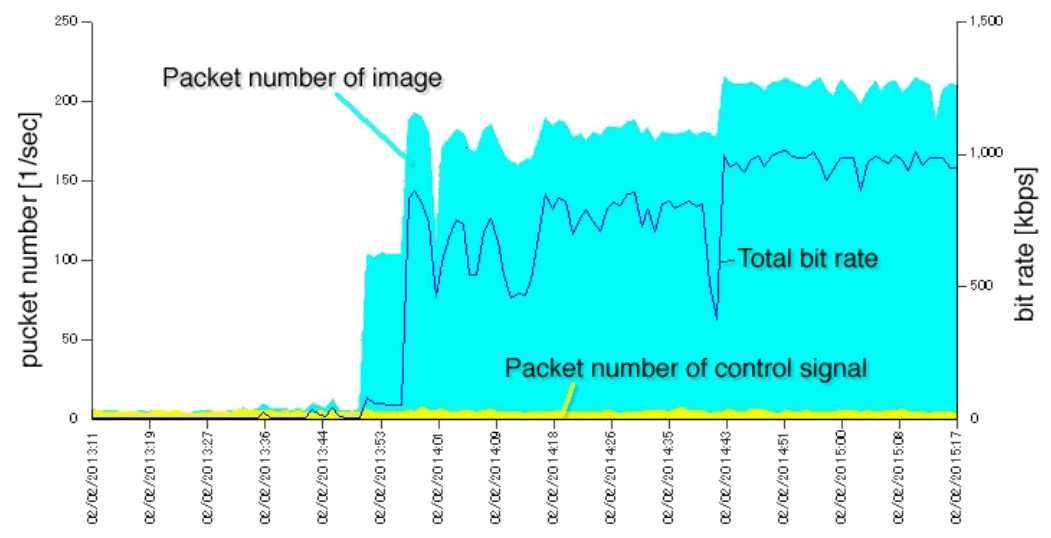

Fig. 6. Time variation of packet number and bit rate during the experiment. Total bit rate was at most $1 \mathrm{Mbps}$, so that this system can be adopted using an ordinary fast digital network

\section{Conclusions}

We have experimented with a wireless tele-echography system to control our echographic diagnosis robot. The examiner could obtain an echogram of a patient at a remote location by control involving a two-axis joystick without any discomfort. The robot never subjected the patient to danger. We have thus confirmed the applicability of tele-echography to telemedicine. This system could be used for remote patients who are living in villages lacking appropriate medical care, or isolated islands far from a main hospital. Japan especially has many mountain areas and small islands, so that demand for telemedicine and telecare is high. Although this system required an assistant on the remote side to place the robot in position, we aim to develop a new system where no personnel are involved. We envisage that this system would be applicable to diagnosis in an emergency situation involving an ambulance or a helicopter, in order to gain time during critical patient transport. Since the advent of satellite systems, international telemedicine and telecare is no longer a dream. We trust that our system will be an effective demonstration of the importance of robotic telemedicine and telecare.

\section{Acknowledgements}

We wish to thank to third grade students of Ehime University, Mr. Sohei Takamori and Miss Toshiko Hidaka who assisted with this experiment. Part of this research was supported by Nakatani Electronic Measuring Technology Association, Japan.

\section{References}

1. K.Masuda, E.Kimura, N.Tateishi and K.Ishihara: "Three Dimensional Motion Mechanism of Ultrasound Probe and its Application for Tele-echography System," Proc. of International Conference of the IEEE Intelligent Robots and Systems (IROS), 2001, Maui, pp.1112-1116 
2. K.Masuda, E.Kimura, N.Tateishi and K.Ishihara: "Construction of 3D Movable Echographic Diagnosis Robot and Remote Diagnosis via Fast Digital Network," Proc. of 23rd Annual International Conference of the IEEE Engineering in Medicine and Biology Society (EMBS), 2001, Istanbul, CD-ROM

3. P.Abolmaesumi, S.E.Salcudean, W.H.Zhu, S.P.DiMaio and M.R.Sirouspour: "A User Interface for Robot-Assisted Diagnostic Ultrasound," Proc. of IEEE Int'1 Conf. on Robotics and Automation, pp.1549-1554, 2001, Seoul, CD-ROM

4. R.P.Goldberg, M.Dumitru, R.H.Taylor and D.Stoianovici: ”A Modular Robotic System for Ultrasound Image Acquisition," Proc. of Medical Image Computing and ComputerAssisted Intervention (MICCAI), 2001, Utrecht, pp.1430-1432

5. M.Mitsuishi, S.Warisawa, T.Tsuda, T.Higuchi, N.Koizumi, et al.: "Remote Ultrasound Diagnostic System," Proc. of IEEE Int'l Conf. on Robotics and Automation, pp.1567-1574, 2001, Seoul, CD-ROM

6. A.V.Gonzales, P.Cinquin, J.Troccaz, A.Guerraz, B.Hennion, et al.:"TER: A System for Robotic Tele-Echography," Proc of Medical Image Computing and Computer-Assisted Intervention (MICCAI), 2001, Utrecht, pp.326-334

7. HORB http://horb.etl.go.jp/horb

8. S.Hirano, Y.Yasu, and H.Igarashi: "Performance Evaluation of Popular Distributed Object Technologies for Java," Proc. of ACM 1998 Workshop on Java for High-performance Network Computing, 1998

9. E.Kimura, K.Matsubara, K.Masuda, N.Tateishi and K.Ishihara: "Remote fetal cardiotocograph watching system with mobile telephone," Proc. of 10th World Congress on Medical Informatics, 2001, London, CD-ROM 